\title{
Kinetic Modeling of Plastic Waste Pyrolysis in a Laboratory Scale Two-stage Reactor
}

To get the formatted version, please use the following DOI: 10.1016/B978-0-444-64235-6.50064-4.

\section{Citation:}

MLA Till, Zoltán, et al. "Kinetic Modeling of Plastic Waste Pyrolysis in a Laboratory Scale TwoStage Reactor." Computer Aided Chemical Engineering. Vol. 43. Elsevier, 2018. 349-354.

APA Till, Z., Varga, T., Sója, J., Miskolczi, N., \& Chován, T. (2018). Kinetic Modeling of Plastic Waste Pyrolysis in a Laboratory Scale Two-Stage Reactor. In Computer Aided Chemical Engineering (Vol. 43, pp. 349-354). Elsevier.

ISO TILL, Zoltán, et al. Kinetic Modeling of Plastic Waste Pyrolysis in a Laboratory Scale Two-

690 Stage Reactor. In: Computer Aided Chemical Engineering. Elsevier, 2018. p. 349-354. 


\title{
Kinetic Modeling of Plastic Waste Pyrolysis in a Laboratory Scale Two-stage Reactor
}

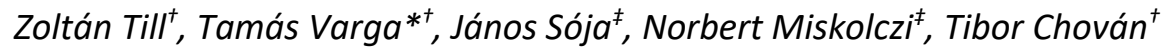 \\ ${ }^{\dagger}$ Department of Process Engineering, University of Pannonia, 10 Egyetem Street, Veszprém H-8200, \\ Hungary \\ ${ }^{\ddagger}$ University of Pannonia, MOL Department of Hydrocarbon and Coal Processing, 10, Egyetem Street, \\ H-8200 Veszprém, Hungary \\ *Email: vargat@fmt.uni-pannon.hu
}

Pyrolysis of waste polymers looks attractive way for their transforming into valuable hydrocarbons. Due to the high number of reactions, the detailed kinetic modeling of the pyrolysis process is often not feasible. Hence the so-called lumping technique is often used to study these complex systems. In this study, the pyrolysis reactions were modeled in a two stage laboratory scale batch reactor using five component lumps. For pyrolysis the mixtures of real plastic waste were used. Kinetic parameters were identified and the model results were compared to the experimental results. It was found that the suggested model can properly describe the dynamic behavior of the reactor under semi batch conditions.

Keywords: discrete lumping, energy from waste, fuel production, kinetic identification, recycling 


\section{Introduction}

Pyrolysis has growing interest in terms of waste recycling. Both biomass (Kim-orba et al., 2015) and petroleum based polymer waste can be transformed into valuable feedstocks for petrochemical sector and refinery. Pyrolysis is a versatile process with a wide product range that can be easily varied with reaction temperature and residence time (Onwudili et al., 2009).

Kinetic modeling of pyrolysis can be challenging due to the various composition of feedstocks and the high complexity of decomposition reactions. The complex network approach is a more sophisticated method to analyze the pyrolysis process. Given that sufficient a priori information is available, the detailed reaction network can be generated automatically; nevertheless the kinetic parameter identification requires some simplification in the model (e.g. the assumption that similar reactions have similar rate coefficients) (Zang et al., 2015). The incremental model identification (IMI) approach can also be applied when the model has a high number of parameters to be identified in case of that the main problem is decomposed into a sequence of subtasks easier to handle individually; for example determine the extent of each reaction in a tubular reactor first then identify the relevant kinetic parameters in each section (Bhatt and Visvanathan, 2015). However, to use this method, concentrations of all components need to be measured along reactor length.

Discrete lumping is a straightforward yet powerful method to analyze complex processes and identify reliable kinetic parameters for a specific process. There are numbers of previous studies (e.g. Ding et al., 2012, Artetxe et al., 2014) dealing with discrete lumping methods, yet the majority of these solutions deal with batch reactors that are extensively used extensively in laboratory scale. Therefore the potential application of discrete lumping for semi batch processes has been investigated. The aim of our study is to identify the kinetic parameters of a lumped reaction network suitable for the thermal pyrolysis of real plastic waste.

\section{Experimental setup}

Plastic waste pyrolysis was carried out in a laboratory scale two-stage reactor at atmospheric pressure. $50 \mathrm{~g}$ of shredded and crashed real plastic waste was charged into the $1^{\text {st }}$ reactor. To ensure inert atmosphere nitrogen was used. Temperature was maintained at $436 / 455 / 471{ }^{\circ} \mathrm{C}$ in the $1^{\text {st }}$ reactor and at $395{ }^{\circ} \mathrm{C}$ in the $2^{\text {nd }}$ reactor. The mixture of decomposed hydrocarbons obtained from $2^{\text {nd }}$ reactor was driven through a water cooled tube-in-tube heat exchanger, where condensable was transformed into liquid phase. During the pyrolysis, samples of liquid products were collected at given intervals, and then were analyzed by gas chromatography. The volume of gaseous products was measured using a gas flow meter.

\section{Reactor Model}

For kinetic studies, a reaction network was proposed consisting five lumps and nine first-order reactions (Figure 1). The model was developed using a discrete lumping approach. Plastic waste feedstock $(P)$ decomposes into lighter pseudocomponents, namely heavier liquid $(L+)$, lighter liquid ( $L-$ ) and pyrolysis gas $(G)$. These lumps were characterised based on the average carbon number of the compounds forming them ( $L+: 15-30, L-: 6-15, G: 0-6)$. The coke is formed during the decomposition of the feedstock and the $L+/ L-$ lumps as well. At the high temperature of the first reactor, $P$ and $C$ are considered to be quasi solid (immobile) and the other pseudocomponents as gaseous (mobile). The mass transfer between the two phases were regarded as instantaneous, in other words, L+, L- and G 
are only present in gas phase while $\mathrm{P}$ and $\mathrm{C}$ form the solid phase. Nitrogen from reactor flush was considered as a standalone component aside from pyrolysis gas.

The rate of the $i^{\text {th }}$ reaction in the $j^{\text {th }}$ reactor is described as a function of the mass concentration of the reactant (with the weight of the specific pseudocomponent divided by the volume of the appropriate phase) (Eq (1)). Reaction rate coefficients were assumed to have Arrhenius-type temperature dependencies (Eq (2)).

$$
\begin{aligned}
& r_{i}^{j}=k_{i}\left(T^{j}\right) \cdot \frac{m_{r}^{j}}{V_{p}^{j}} \\
& k_{i}=k_{0, i} \cdot \exp \left(-\frac{E_{a, i}}{R \cdot T^{j}}\right)
\end{aligned}
$$

Component sources were calculated from reaction rates and the stoichiometric matrix of the reaction network (Eq (3)). In order to maintain the mass balance, reaction rates were multiplied by the volume of the phase in that the reaction takes place (Eq (4)).

$$
\begin{aligned}
& \underline{R}^{j}=\underline{v} \cdot \underline{r}^{j} \\
& r_{i}^{\prime j}=V_{p}^{j} \cdot r_{i}^{j}
\end{aligned}
$$

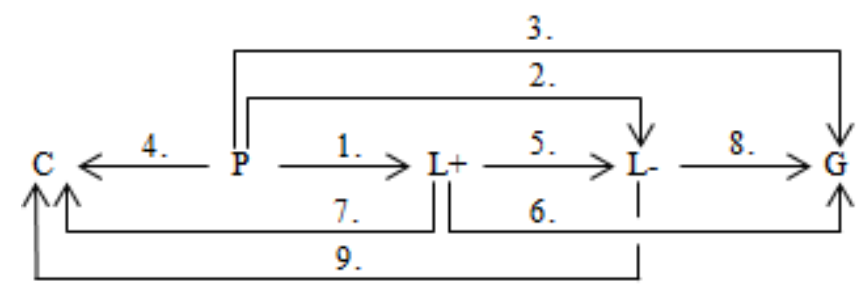

Figure 1. Suggested reaction network consisting five component lumps and nine lumped reactions.

Both reactors were considered to be ideally mixed given the small volumes and low residence time. The change in mass of component $c$ is the result of the difference between the inflow and outflow rates as well as its source from $\mathrm{Eq}(3)$ :

$$
\frac{d m_{c}^{j}}{d t}=\dot{m}_{c}^{j, \text { in }}-\dot{m}_{c}^{j, \text { out }}+R_{c}^{j}
$$

For components $\mathrm{P}$ and $\mathrm{C}$, flow rate calculation can be omitted because of immobility. In case of the $1^{\text {st }}$ reactor, gaseous component inflow rates are equal to zero except for the nitrogen flush while the inlet of the $2^{\text {nd }}$ reactor is equal to the outlet of the $1^{\text {st }}$ vessel. Reactor outflow (Eq (6)) is a result of three independent phenomena:

- components are formed or depleted in the reactor;

- component mass is increased because of inflow;

- gas phase volume increases with the decrease of the solid phase.

$$
m_{c, g}^{j, o u t}=\left(\sum_{k} \frac{R_{k}^{j}}{M_{k}}+\sum_{k} \frac{m_{k}^{j, \text { in }}}{M_{k}}+\frac{d n_{g}^{j}}{d t}\right) \cdot x_{c, g}^{j} \cdot \bar{M}_{c}
$$


Assuming that the behavior of gaseous phase follows the ideal gas law, at constant pressure and temperature the molar mass of substance changes with the gas phase volume as described in Eq (7):

$$
\frac{d n_{g}^{j}}{d t}=-\frac{p}{R \cdot T^{j}} \cdot\left[\frac{d}{d t}\left(\frac{m_{P}}{\rho_{P}}\right)+\frac{d}{d t}\left(\frac{m_{C}}{\rho_{C}}\right)\right]
$$

The aim of our study is to determine the reaction kinetic parameters defined in Eq (2) to model and predict thermal degradation of polymer waste. The objective is to minimize the difference between measured and calculated weight fractions (Eq (8)), in that square error between measurement $(m)$ and calculation $(c)$ is summarized for three temperature $(T)$ levels, five pseudocomponents (comp) and all measurement points $(p)$ (one for $\mathrm{P}, \mathrm{C}$ and $\mathrm{G}$; four to six for $\mathrm{L}+$ and $\mathrm{L}-$ ):

$$
f\left(\underline{x}^{n}\right)=\sum_{T} \sum_{\text {comp }} \sum_{p}\left(w_{m}-w_{c}\right)^{2}
$$

Decision variables in Eq (8) are expressed in Eq (9-10) and include the pre-exponential factors and activation energies for each reaction, normalized between 0 and 1 for better convergence. Values of lower and upper bounds summarized in Table 2 were chosen as a result of a set of preliminary studies regarding the values of rate coefficients at individual temperature levels.

$$
\begin{aligned}
& \underline{x}^{n}=\left[\begin{array}{ll}
\left(k_{0}^{n}\right)^{T} & \left(E_{a}^{n}\right)^{T}
\end{array}\right]^{T} \\
& x^{a c t}=x^{n} \cdot\left(u_{b}-l_{b}\right)+l_{b}
\end{aligned}
$$

The dynamic model of the laboratory scale two-stage pyrolysis reactor was implemented and solved in MATLAB R2011b. The reactor model was solved using the variable-step, variable-order (VSVO) solver based on the numerical differentiation formulas called ode15s (Shampine and Reichelt, 1997). For solving the optimization problem, the NOMAD software package was used that implements the Mesh Adaptive Direct Search (MADS) algorithm and is a well suited solver for derivative-free optimization (Le Digabel, 2011). It also has a MATLAB interface available that can be called directly from the OPTI Toolbox (Currie and Wilson, 2012).

\section{Results and discussion}

Measured and calculated mass fractions are shown in Figure 2 as a function of time. In case of liquid and gas products ( $L+, L-$ and $G$ ) the mass fraction was calculated as a quotient of the amount that has left the $2^{\text {nd }}$ reactor and the initial charge; while in case of $P$ and $C$, the amounts inside the two reactors were taken into consideration. The resulting curves appear to indicate that the suggested reaction network can properly describe the concentration changes taking place during polymer waste pyrolysis. Curve fitting for both liquid fractions are mostly the same and is acceptable within reason. The model predicts the final mass of $\mathrm{G}$ and $\mathrm{C}$ accurately, while predicted remaining $\mathrm{P}$ amount is fairly high. 

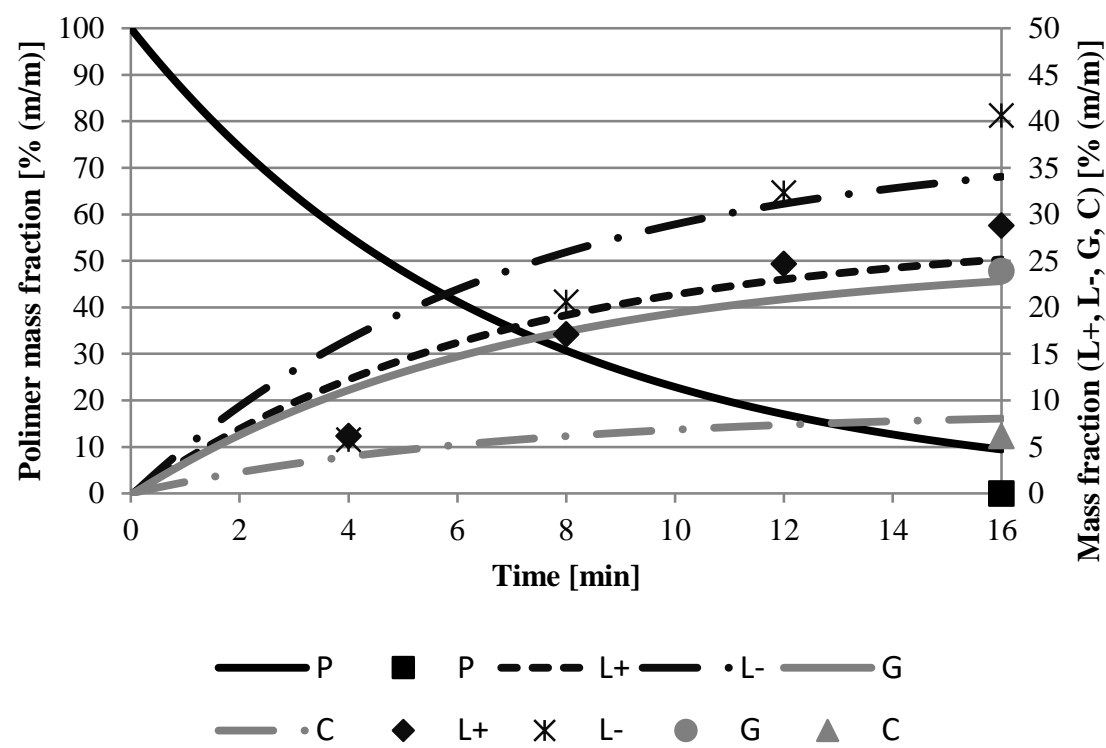

Figure 2. Pseudocomponent mass fractions at $455^{\circ} \mathrm{C}$ - experimental (markers) and model (lines)

To express the reliability of the identified kinetic parameters quantitatively, the root of mean square errors between measured data and model results for each lump and the complete data set were calculated and are listed in Table 1 . Overall, the lowest values were obtained for $436{ }^{\circ} \mathrm{C}$ reactor temperature. The identified model gives reasonable estimation for $\mathrm{G}$ and $\mathrm{C}$; on the other hand, it tends to overestimate the remaining amount of $\mathrm{P}$ at the end of the run. Estimated values of $\mathrm{L}+$ and $\mathrm{L}-$ are in reasonable agreement with the measured data; however, as shown in Figure 2, the actual formation of these components is slightly slower at the beginning than predicted.

Table 1. Root-mean-square error (RMSE) between measured and calculated data

\begin{tabular}{|l|r|r|r|r|r|r|}
\hline & P & \multicolumn{1}{|l|}{ L+ } & L- & G & C & Lump aggregated \\
\hline $436{ }^{\circ} \mathrm{C}$ & $7.0 \%$ & $1.9 \%$ & $2.6 \%$ & $2.8 \%$ & $0.9 \%$ & $2.8 \%$ \\
\hline $455{ }^{\circ} \mathrm{C}$ & $9.4 \%$ & $3.8 \%$ & $6.9 \%$ & $1.1 \%$ & $1.9 \%$ & $5.6 \%$ \\
\hline $471{ }^{\circ} \mathrm{C}$ & $5.3 \%$ & $3.2 \%$ & $3.8 \%$ & $1.3 \%$ & $0.2 \%$ & $3.4 \%$ \\
\hline $\begin{array}{l}\text { Temperature } \\
\text { aggregated }\end{array}$ & $7.4 \%$ & $2.9 \%$ & $4.5 \%$ & $1.9 \%$ & $1.2 \%$ & $4.0 \%$ \\
\hline
\end{tabular}


The identified kinetic parameters for the suggested reaction network as well as the values of reaction rate coefficients at $455^{\circ} \mathrm{C}$ are listed in Table 2. It is visible that the rate coefficients for the first four reactions are significantly higher than for the latter five; this could indicate that the formation of products during polymer waste pyrolysis happens simultaneously rather than following a consecutive pattern. This is also supported by reaction networks suggested previously in literature (Ceamanos et al., 2002, Al-Salem and Lettieri, 2010).

Table 2. Lower and upper bounds, identified kinetic parameters and reaction rate coefficients for the specified reactions

\begin{tabular}{|l|r|r|r|r|r|r|r|}
\hline \multirow{2}{*}{ Reaction } & \multicolumn{3}{|c|}{$\mathrm{k}_{0}\left[\mathrm{~s}^{-1}\right]$} & \multicolumn{2}{|c|}{$\mathrm{E}_{\mathrm{a}}\left[\mathrm{kJ} \mathrm{mol}^{-1}\right]$} & \multirow{2}{*}{$\mathrm{k}$ at $455{ }^{\circ} \mathrm{C}\left[\mathrm{s}^{-1}\right]$} \\
\cline { 2 - 6 } & \multicolumn{1}{|c|}{$\mathrm{I}_{\mathrm{b}}$} & \multicolumn{1}{c|}{$\mathrm{I}_{\mathrm{b}}$} & actual & \multicolumn{1}{|c|}{$\mathrm{l}_{\mathrm{b}}$} & \multicolumn{1}{c|}{$\mathrm{u}_{\mathrm{b}}$} & actual & \\
\hline $\mathrm{P} \rightarrow \mathrm{L}+$ & $1.00 \cdot 10^{16}$ & $1.00 \cdot 10^{16}$ & $1.23 \cdot 10^{16}$ & 10.0 & 500 & 268.37 & $6.87 \cdot 10^{-4}$ \\
\hline $\mathrm{P} \rightarrow \mathrm{L}-$ & $1.00 \cdot 10^{19}$ & $1.00 \cdot 10^{19}$ & $7.62 \cdot 10^{19}$ & 10.0 & 500 & 319.40 & $9.31 \cdot 10^{-4}$ \\
\hline $\mathrm{P} \rightarrow \mathrm{G}$ & $1.44 \cdot 10^{2}$ & $1.44 \cdot 10^{2}$ & $9.94 \cdot 10^{2} \mathrm{E}$ & 10.0 & 500 & 86.46 & $6.24 \cdot 10^{-4}$ \\
\hline $\mathrm{P} \rightarrow \mathrm{C}$ & $1.20 \cdot 10^{12}$ & $1.20 \cdot 10^{12}$ & $1.07 \cdot 10^{13}$ & 10.0 & 500 & 232.67 & $2.18 \cdot 10^{-4}$ \\
\hline $\mathrm{L}+\rightarrow \mathrm{L}-$ & $1.44 \cdot 10^{-2}$ & $1.44 \cdot 10^{-2}$ & $1.24 \cdot 10^{-1}$ & 17.3 & 864 & 229.71 & $4.10 \cdot 10^{-18}$ \\
\hline $\mathrm{L}+\rightarrow \mathrm{G}$ & $1.66 \cdot 10^{-1}$ & $1.66 \cdot 10^{-1}$ & 1.18 & 14.4 & 720 & 386.17 & $2.34 \cdot 10^{-28}$ \\
\hline $\mathrm{L}+\rightarrow \mathrm{C}$ & $7.68 \cdot 10^{-4}$ & $7.68 \cdot 10^{-4}$ & $1.37 \cdot 10^{-3}$ & 14.4 & 720 & 215.74 & $4.57 \cdot 10^{-19}$ \\
\hline $\mathrm{L}-\rightarrow \mathrm{G}$ & $6.40 \cdot 10^{-1}$ & $6.40 \cdot 10^{-1}$ & 3.94 & 14.4 & 720 & 208.21 & $4.56 \cdot 10^{-15}$ \\
\hline $\mathrm{L}-\rightarrow \mathrm{C}$ & 1.15 & 1.15 & 6.95 & 17.3 & 864 & 393.89 & $3.85 \cdot 10^{-28}$ \\
\hline
\end{tabular}

\section{Conclusions and Future Work}

In this work, kinetic parameters of a lumped reaction system consisting five pseudocomponents and nine reactions were identified from experimental data obtained in a laboratory scale two-stage semi batch reactor. In general, the proposed model can effectively describe the concentration changes in the system, while also fast and slow reaction routes can be distinguished; this fact might give room for further model reduction. Future work may consider the non-isothermal behaviour of the reactor, i.e. taking the heat-up period of measurements into consideration. The proposed method can be applied for catalytic pyrolysis as well, for instance the effect of different catalysts on individual reactions can be investigated. 


\section{Acknowledgements}

We acknowledge the financial support of Széchenyi 2020 under the GINOP-2.3.2-15-2016-00053. János Sója and Norbert Miskolczi acknowledge for the financial support of Széchenyi 2020 under the EFOP3.6.1-16-2016-00015.

\section{References}

S. M. Al-Salem and P. Lettieri, 2010. Kinetic study of high density polyethylene (HDPE) pyrolysis, Chemical Engineering Research and Design, 88 (12), 1599-1606.

M. Artetxe, G. Lopez, M. Amutio, J. Bilbao, M. Olazar, 2014. Kinetic modelling of the cracking of HDPE pyrolysis volatiles on a HZSM-5 zeolite based catalyst, Chemical Engineering Science, 116 (Suppl. C), 635-644.

N. Bhatt and S. Visvanathan, 2015. Incremental Kinetic Identification based on Experimental data From Steady-state Plug Flow Reactors, in K. V. Gernaey, J. K. Huusom, és R. Gani, (Eds), Computer Aided Chemical Engineering, 37 (Suppl. C), 593-598, Elsevier, 2015.

J. Ceamanos, J. F. Mastral, A. Millera, M. E. Aldea, 2002. Kinetics of pyrolysis of high density polyethylene. Comparison of isothermal and dynamic experiments, Journal of Analytical and Applied Pyrolysis, 65 (2), 93-110.

J. Currie and D. Wilson, 2012. OPTI: Lowering the Barrier Between Open Source Optimizers and the Industrial MATLAB User, In Foundations of Computer-Aided Process Operations, Savannah, GA, USA, Jan. 8-13, 2012., N. Sahinidis, J. Pinto (Eds), Elsevier: Toronto, 2012.

F. Ding, L. Xiong, C. Luo, H. Zhang, X. Chen, 2012. Kinetic study of low-temperature conversion of plastic mixtures to value added products, Journal of Analytical and Applied Pyrolysis, 94 (Suppl. C), 83-90.

Z. Fang, T. Qiu, B. Chen, 2015. Analyzing and Modeling Ethylene Cracking Process with Complex Networks Approach, in K. V. Gernaey, J. K. Huusom, és R. Gani (Eds), Computer Aided Chemical Engineering, 37 (Suppl. C), 407-412, Elsevier, 2015.

K. Im-orba, L. Simasatitkul, A. Arpornwichanop, 2015. Performance Analysis and Optimization of the Biomass Gasification and Fischer-Tropsch Integrated Process for Green Fuel Productions, in K. V. Gernaey, J. K. Huusom, R. Gani (Eds), Computer Aided Chemical Engineering, 37 (Suppl. C), 275-280, Elsevier, 2015.

S. Le Digabel, 2011. Algorithm 909: NOMAD: Nonlinear optimization with the MADS algorithm, ACM Transactions on Mathematical Software, 37 (4), 44.

J. A. Onwudili, N. Insura, P. T. Williams, 2009. Composition of products from the pyrolysis of polyethylene and polystyrene in a closed batch reactor: Effects of temperature and residence time, Journal of Analytical and Applied Pyrolysis, 86 (2), 293-303.

L. Shampine and M. Reichelt, 1997. The MATLAB ODE Suite, SIAM Journal on Scientific Computing, 18 (1), 1-22. 